\title{
HUMAN GAIT ANALYSIS FOR HIP IMPLANTED PERSONS
}

\author{
Diana L. Cotoros and Mihaela I. Baritz \\ Transilvania University from Brasov \\ 29, Eroilor Av., 500036 Brasov, Romania \\ E-mail: dcotoros@unitbv.ro, mbaritz@unitbv.ro
}

\author{
KEYWORDS \\ Biomechanics, gait, human body implant
}

\begin{abstract}
In this paper, the research team presents some aspects concerning the design of a methodology and a human gait analysis system for persons who suffered a locomotor disfunctionality and recovered by means of implants. Thus, in the first part of the paper we analyze theoretical aspects connected to the human body biomechanics in the process of normal gait but also those concerning the emphasis of locomotor disfunctionalities. In the second part of the paper, the recording methodology for gait dynamic parameters and the selected cases are presented. In the last part we show the results and conclusions obtained based on the proposed methodology.
\end{abstract}

\section{INTRODUCTION}

Prosthesis and/or orthesis use on neuro-motor deficient persons is absolutely necessary for many patients with bones and joints, ligament, muscular, vascular, peripherical and/or central neural deficiencies. It plays an important part within the rehabilitation measures for these patients. Prosthesis is a mean of replacing a missing anatomical segment (congenital or taken). It may be for esthetical reasons (for limbs or eye-balls), for functional mechanical purpose or using biocurrents. Orthesis is used to increase the functionality of an already existing anatomical segment, which proves to be deficient from morpho-functional point of view. It is highly important that the prosthesis or orthesis is applied in due time, as soon as possible, first on temporary basis and then on a long term basis or for the rest of the patient's life. The gait re-education is required for all forms of locomotor deficient persons.

Kineto- therapy is an important source, offering the best possible recovery to the patients. Exercises should be carefully dosaged in order to avoid the human subjects' fatigue. For better and long term efficiency they need to use relaxation techniques, simple balances and rhythmical techniques, which stimulate the vestibular system in order to obtain the general relaxation of the body muscles. The rocking-chair use reduces the muscular tonus and decreases the stiffness, as well as the rhythmical and slow roll-over the mattress have the same effect. The upside down head posture, such as the patient resting with the ventral area on a big ball produces a general relaxation by activating some medullar centers - methods that should not take too long in order to avoid the change of other physiological parameters. Thus, a series of respiratory exercises are performed with the purpose of increasing the thoracic and diaphragm mobility, while the deep and slow breaths lead to the mitigation of muscular stiffness. In order to obtain optimal results, the active and passive mobilizations must be made several times a day, thus recommending techniques of neural-muscular and prioceptive facilitation: contraction - relaxation, passive prolonged stretching.

At the same time, the exercises should be correlated with the self-caring functions because they raise the motivation and reduce some exercise abandoning manifestations.

During performing the recovery or training actions, some verbal commands even accompanied by musical rhythms are very useful, as well as big mirrors closely positioned may help to the motions regularity.

\section{MOVEMENT ANALYSIS OF HUMAN BODY WITH GAIT AND POSTURAL DISABILITIES}

During the analysis of the human motions locomotor disabilities, we started from developing a synthetic concept model, which emphasized the causes and the main milestones in measuring and assessment

In this respect we found that the locomotor disfunctionalities should be treated as substantial changes of the human body kinematics, reflected to the level of reaction forces magnitude and direction between the locomotor system and the ground.

Fig. 1 presents a synthetic model of key factors that contribute to quasi-static and dynamic postural control. This model includes feed-forward and feedback control functions. The anticipated postural disturbances, involuntarily known by the subject arise from the forces generated during the performance of voluntary motion, or in anticipation of destabilizing external forces.

This type of postural adjustment, referred to as anticipatory, involves estimation of the magnitude and direction of postural disturbance and initiation of a motor program by the central nervous system. The motor program is a set of commands that is selected based on an internal or previous model of the task to be performed. Once initiated, the motor program is executed in an open loop manner, but with the potentially processing delays inherent in feedback control. Ideally, the motor program activates the 
musculature that produces a set of appropriately scaled and timed pre-emptive muscle forces and joint moments that precede and deny the anticipated postural disturbance. During feedback control, a postural disturbance, the origin of which may be either internal or external, causes a change in body posture or movements. If the change in kinematics exceeds some threshold value to which the central nervous system has assigned importance, a corrective postural response will be generated.

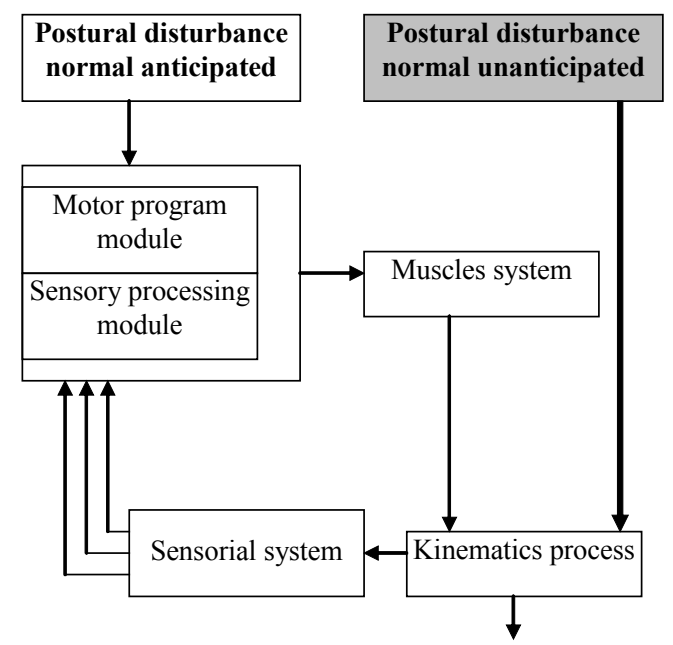

Fig.1. Data model of human static or dynamic posture

The changes in the kinematics process stimulate visual, vestibular, and somatic-sensorial system, sensors that transmit the information to the central nervous system. Information processing by the sensorial system involves comparisons for detecting the state of the body into a desired state. In an ideal configuration, if the difference between the detected and desired states, that is, an error signal, is of sufficient magnitude, a corrective postural response consisting of muscle forces and joint moments will be executed to obtain the final response. The muscle forces and joint moments affect body kinematics, generating a new set of sensor information signals and another loop of the feedback process is initiated in this model.

\section{EXPERIMENTAL SETUP}

Within the researches concerning the hip implanted human factor gait analysis we developed a flexible and interconnected system consisting of a Kistler force plate, computerized data acquisition and processing system, high speed video camera, Fasted type with 500 frames/sec and licensed software for human gait modeling LifeMode.

The investigation conditions were established at the initial temperature, humidity and atmospheric pressure parameters, within normal limits.

The researched human subject was initially investigated and the physiological parameters such as: pulse, temperature, blood pressure, weight and height were recorded during different times of the day, when the investigations took place. The measured physiological data upon the human subject are the following: height $1,6 \mathrm{~m}$; weight $55,31 \mathrm{~kg}$; temperature $36,3 \mathrm{C}^{0}$; blood pressure $14,3 / 7,4$; pulse 70 ; medical diagnosis: COTOLOIDITA right hip (7 years old partial prosthesis); recording observations - partial help for climbing up and down in order to preserve balance.

The human subject is female, 74 years old and presents the urge of replacing the previous implanted hip prosthesis.

By help of the previous established procedure we aimed at the assessment of the dynamic parameters of the different gait variants, for which we analyze the human subject, thus accomplishing recording before the surgical intervention, immediately after and one month after, preserving the same investigation methodology.

The analysis of the X-ray images of the human subject reveals functional and position abnormalities and the decision of the medical staff was to replace the implant with a new one adjusted to the new bone configuration.

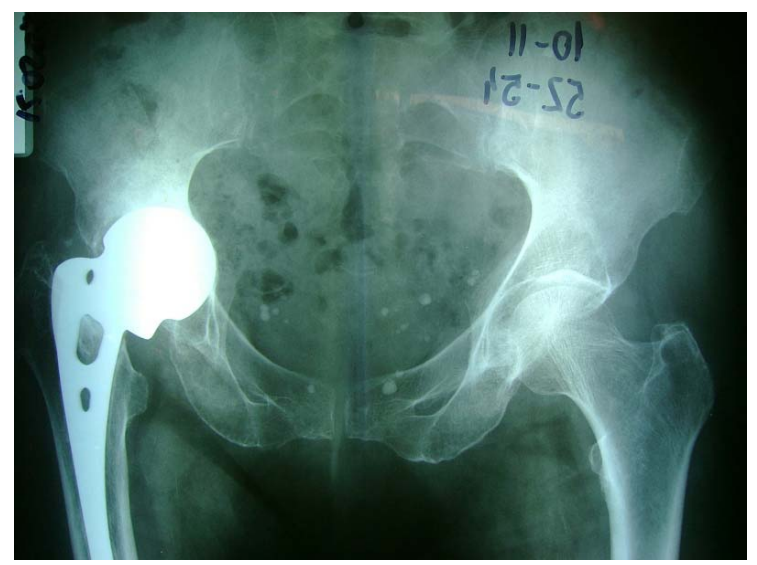

Fig.2. X-ray image of the previous implant

Due to wear and changes of the bone structure, the subject's gait was changed from the time of implant introduction until the investigation time determining an increase of the locomotor malfunction also accompanied by painful manifestations.

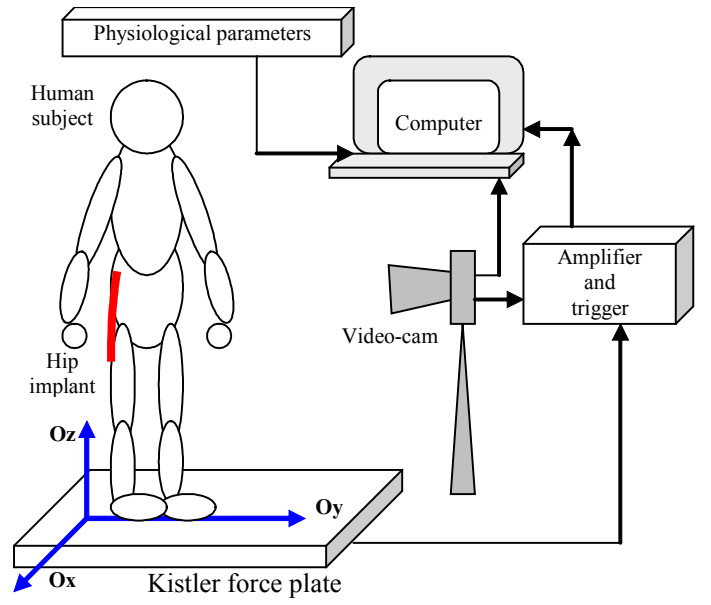

Fig.3. Experimental setup 
Analysis processed by help of the experimental structure proposed for this research and presented in fig. 3 revealed these locomotor malfunctions, allowing the development of a faster and optimized gait rehabilitation procedure.

Using this system we accomplished a series of recordings of the following gait possibilities: normal gait, added step gait, dragged gait, paced gait and backwards gait.

These recording of the gait modes were entirely performed before the implant replacement but also immediately after the surgery, when only one gait mode was possible without external help or support.

After the first recovery period (one month) we repeated the recordings of the gait modes, observing by means of the dynamic parameters analysis of the recording on the force plate, an obvious improvement.

Also the X-ray results prove the regaining hip mobility, these being corroborated with the results obtained from the software modeling and those recording by help of the experimental setup.

\section{RESULTS AND CONCLUSIONS}

The recordings were made in various times of the day before the surgery and the obtained results are presented in the following figures.

The average period of a recording was $10 \mathrm{sec}$ and the motion was performed in two steps, the first one being the leg without an implant.
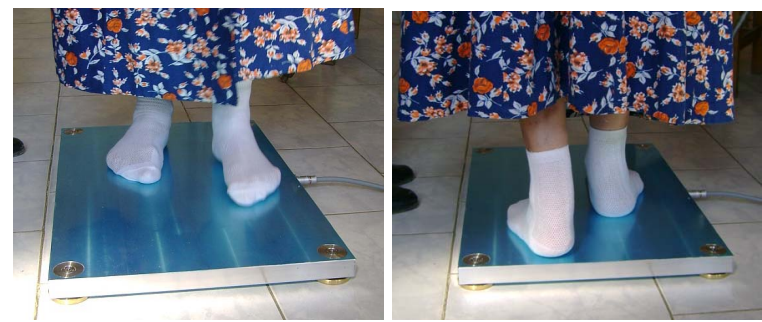

Fig. 4. Human subject on Kistler force plate, normal gait, two steps, starting with left leg

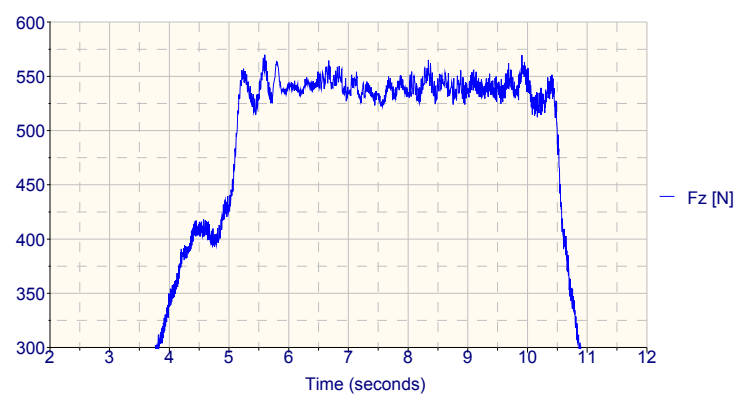

Fig. 5. Fz force graph, normal gait, two steps

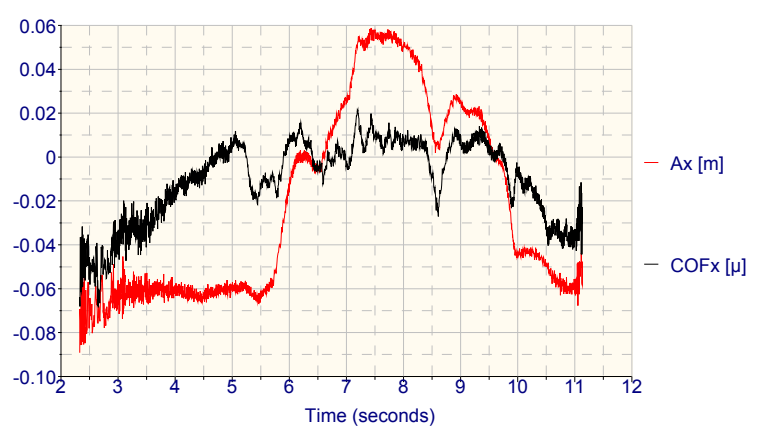

Fig.6. Variation of Ax and COFx in the same recording

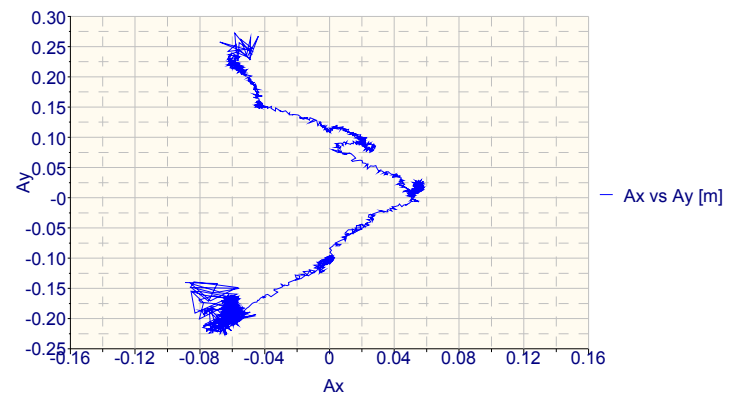

Fig.7. Surface and direction of stability field

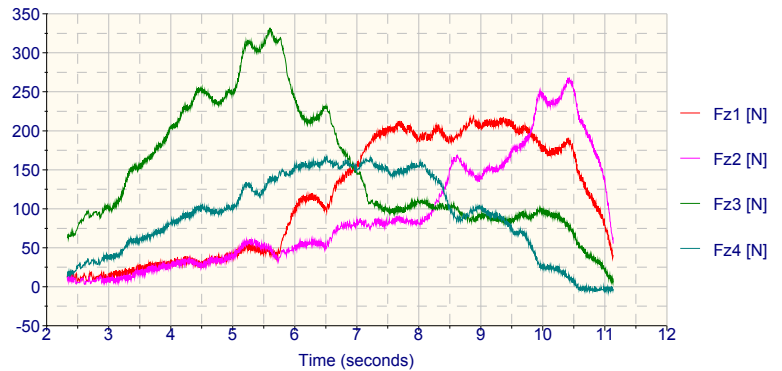

Fig.8. Force values on $\mathrm{Oz}$ direction measured by 4 piezoelectric sensors of Kistler force plate

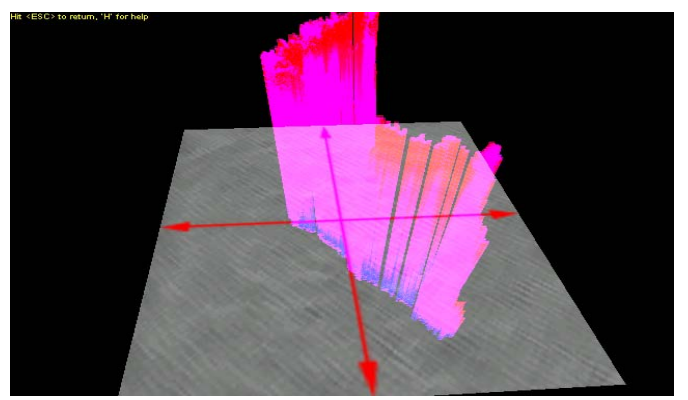

Fig. 9. The measured forces in contact time

As we may notice in the previous figures, the human subject with a 7 years old hip implant presents an important change of the normal gait, the support during gait is mainly made on the left leg, the right one being protected because it produces pain; also a strong values unbalance between the reaction forces on $\mathrm{Oz}$ measured by the 4 piezoelectric sensors (fig.7) and last but not 
least a displacement of an important part of the body weight upon the left leg joint, respectively a displacement of the gravity centre on the support surface with the tendency of falling outside it. (fig.5).

In this respect the surgery - change of hip implant will have to correct the deficiencies and give back the mobility and motility of the locomotor system within normal limits.

Analyzing the other gait modes - added, dragged, paced and backwards we may conclude that in all cases important deviations from the normal limits occur, the subject loses balance and requires support and help during displacement, also claiming pains.

One week after the surgery, the investigations were resumed and the same set of measurements was performed in the same environmental conditions.

In the next figures, the recordings results are presented and we may notice that the displacement period is significantly longer (double), also the values of the force on $\mathrm{Oz}$ changed dramatically, thus emphasizing the post surgery discomfort.

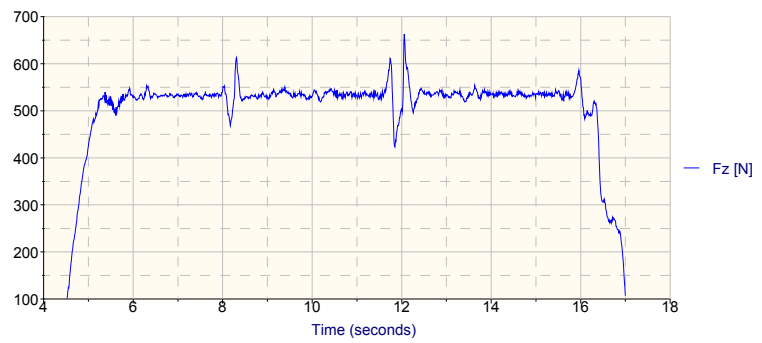

Fig. 10. Fz force graph, normal gait (after 1 week) post surgery, two steps

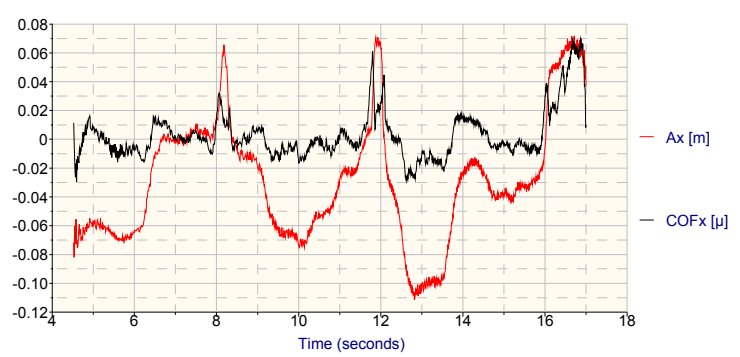

Fig.11. Variation of Ax and COFx in the same recording post surgery

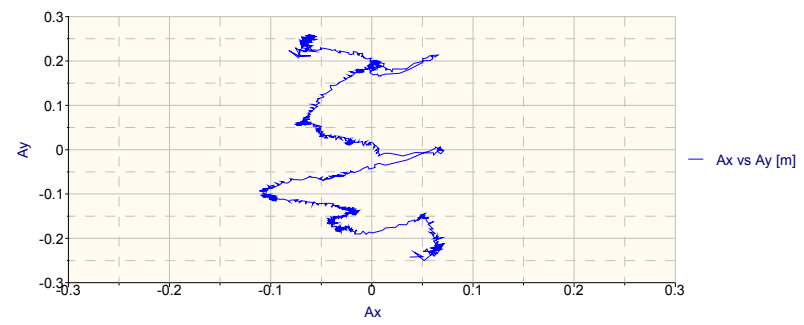

Fig.12. Stability field surface and direction for post surgery situation

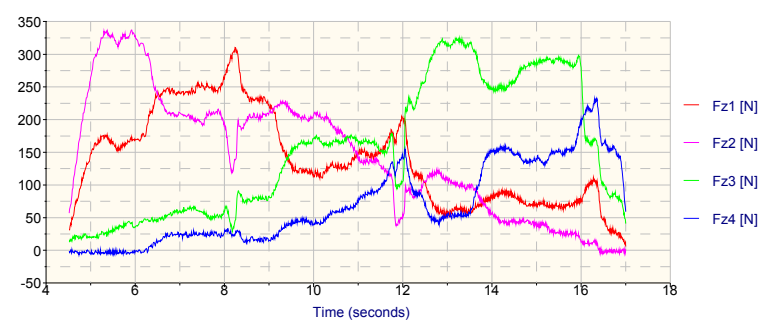

Fig.13. Force values (post surgery) on $\mathrm{Oz}$ direction measured by 4 piezoelectric sensors of force plate

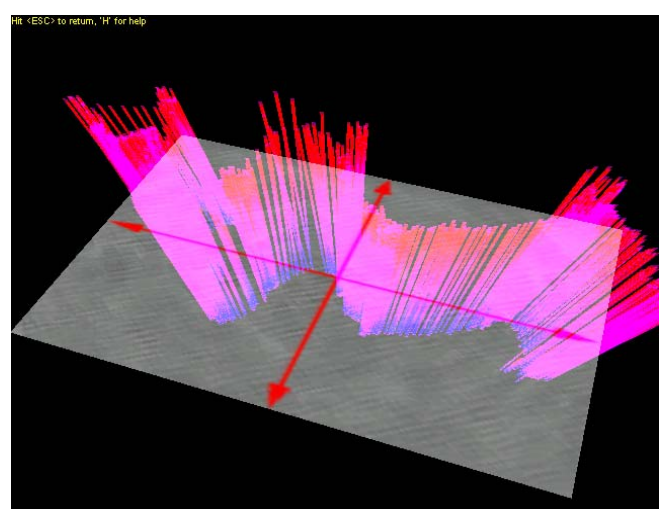

Fig. 14. The measured forces in contact time

The recorded physiological parameters during this intermediate investigation (a week after the surgery) were: weight $54,33 \mathrm{Kg}$; temperature $36,3 \mathrm{C}^{0}$; blood pressure 12/4; pulse: 77 ; medical diagnosis: total hip prosthesis surgery using a cemented prosthesis; with the following observations: climbing up and down the force plate required partial help for preserving balance, while during normal gait the subject moves heavily using a metal frame.

After a six week recovery period, the same subject was investigated using the same experimental structure and the same environmental conditions.
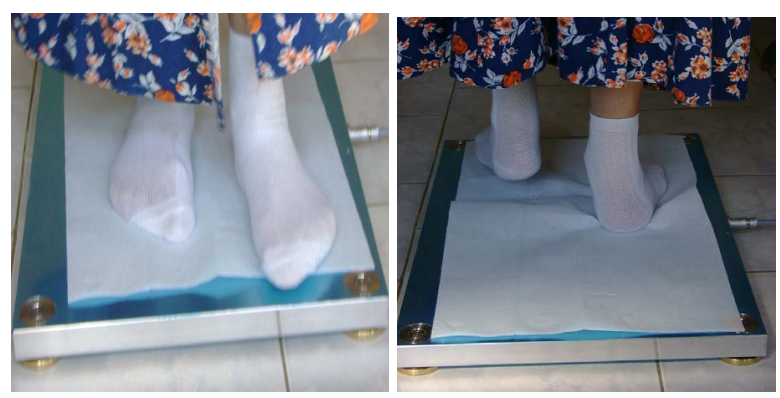

Fig. 15. Human subject (post surgery) on Kistler force plate, normal gait, two steps, starting with left leg

The physiological parameters recorded after six weeks from the surgery, changed as follows: weight $56,7 \mathrm{Kg}$; temeprature $36,3 \mathrm{C}^{0}$; blood pressure $15,5 / 9$ at the beginning of the recordings and 14,8/8,6 at the final of the recordings; pulse 77 and respectively 78 ; 
observations: partial help on climbing up and down the plate for balance preserving, during gait subject still needs sometimes a crutch; there are slight pains but without incurring locomotor discomfort.

Out of the recorded data at this moment we may observe a significant improvement of the subject's normal gait, due to the similarity increase between the graph of the measured reaction force and the normal graph shape.

An eloquent example proving the above stated is represented by the comparative analysis of the $\mathrm{Oz}$ reaction force graph during the dragged gait (because great pains were claimed and previously this type of displacement was used) in three research stages: before, immediately after and six weeks after the surgery.

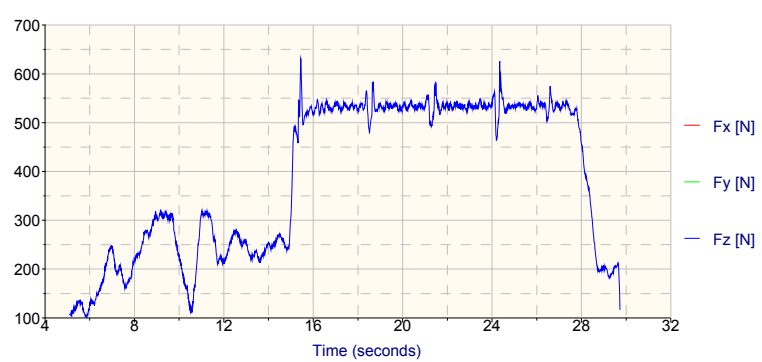

Fig.16. Fz force graph, in dragged gait before surgery

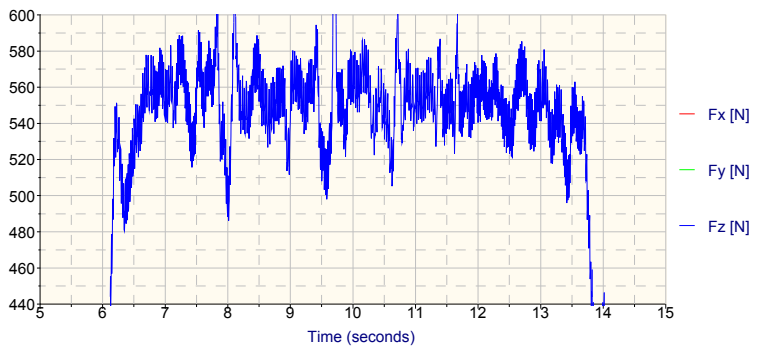

Fig.17. Fz force graph, in dragged gait a week after surgery

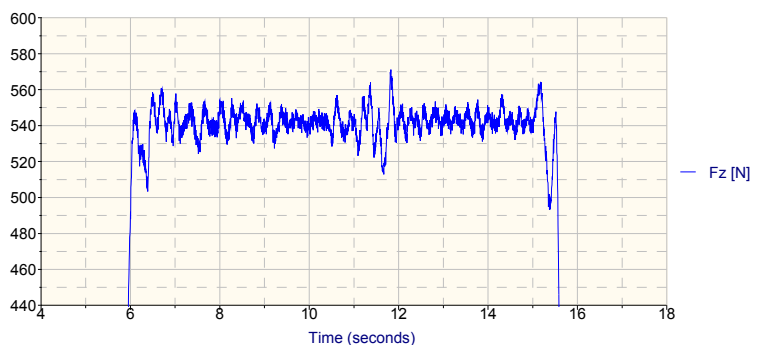

Fig.18. Fz force graph, in dragged gait six weeks after surgery

In this respect we mention that the displacement period for this mode constantly decreased, the subject having an increased mobility and a correct balance.

Also the variations of the reaction force are constant around $540 \mathrm{~N}$ in six weeks post surgery situation, unlike the initial situation when this value was not constant, the variation being between $100 \mathrm{~N}$ and $590 \mathrm{~N}$.

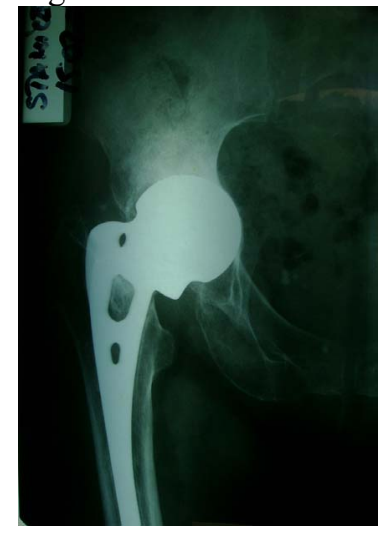

Fig.19. X-ray image of the replaced implant

Using these recordings we could rapidly and optimally asses the recovery stage of the human subject, who gained a better mobility and easier adopted a more balanced posture and firmer displacement.

The used experimental structures is flexible, easy to manipulate and gives the opportunity of creting an objective database for each subject, this way being able to mitigate the locomotor recovery and rehabilitation period of the implanted patients.

At this moment, using the adopted modeling and simulation methods we can accomplish rehabilitation protocols of the person with hip implant and not least assessment strategies for the post-operatory evolution.

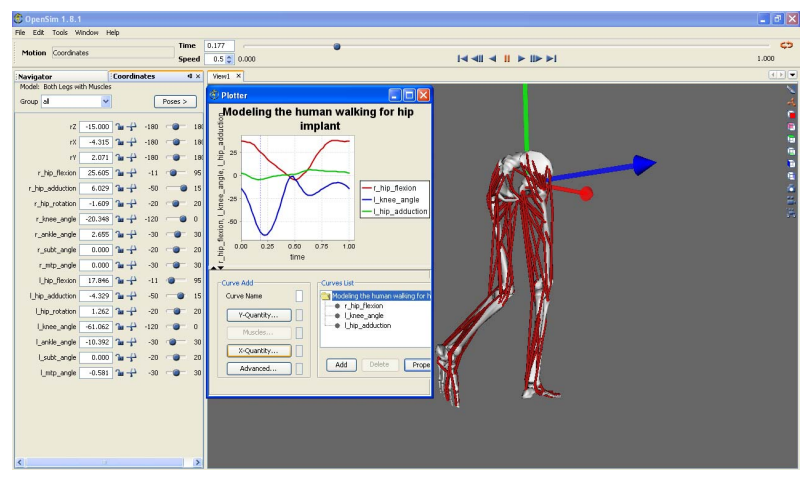

Fig. 20. Use of the software package OpenSim ver.1.8.1. for simulating the post-operatory affliction

The modeling and simulation methodology of the gait afflicted by the surgical intervention is based on the inverse kinematics and dynamics applied to the models offered by the database of the software package OpenSim. Thus, starting form the complex form skeleton - tendon - muscle - joint form the software OpenSim database we introduced on this model, the measured values evaluated by the presented investigation methodology and we obtained the simulations corresponding to the recording moment $(7$ days, respectively 30 days after surgery). The motion equations for the muscle - skeletal system allow the 
calculus of the generalized coordinates accelerations resulted by applying different forces or motion limitations.

$$
\begin{aligned}
& \ddot{\vec{a}}=\vec{M}(\vec{a})^{-1}\left[\vec{F}\left(\vec{a}, \dot{\vec{a}}^{2}\right)+\vec{G}(\vec{a})+\vec{\Gamma}(\vec{a}, \dot{\vec{a}})+\vec{R}(\vec{a}) \vec{f}_{P}+\right. \\
& \left.+\vec{S}(\vec{a}) \vec{f}_{S}(\vec{a}, \dot{\vec{a}})\right\rfloor
\end{aligned}
$$

From the complementary analysis of the obtained data we can observe the similitude between the forces variation measured using the force plate and those modeled using the software package, which allows the concept of a data correlation structure.

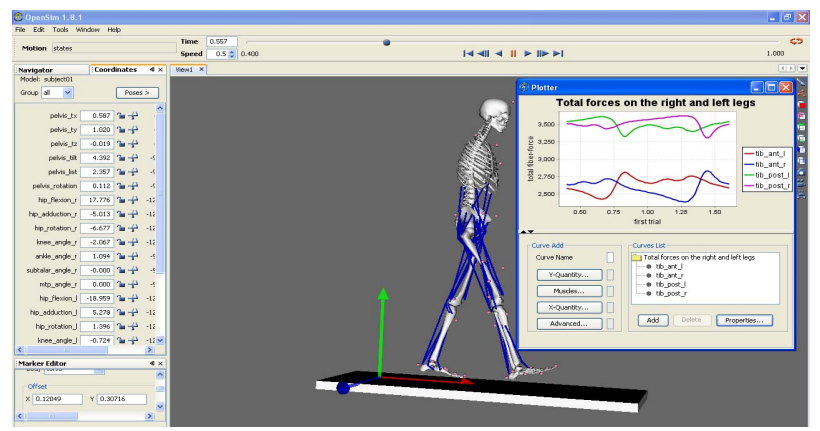

Fig.21. Modeling of the forces action on two feet for a post-operatory patient with hip implant

This structure will allow the specialist doctor to assess and predict the subsequent post-operatory evolution of the patient and also create a fast and efficient recovery strategy.

The modeling and simulation structure, briefly presented for this case represents the subject of a more extended research, which allows the developing of an investigation-assessment-rehabilitation protocol for the hip implant patients.

\section{ACKNOWLEDGMENTS}

Researches are part of Grant A1088, Grant PNII-Idei 722 and 744 with CNCSIS Romania and the investigations were developed using equipment belonging to the Research Platform "SAVAT", University Transylvania Brasov.

We want to thank to our medical staff from Municipal Hospital of Brasov for their useful help.

\section{REFERENCES}

Baritz M., Cotoros D., Cristea L., . "Complex systems for human body biomechanical behavior modeling." Bio-Inspired Computational Methods Used for difficult problems Solving. Development of Intelligent and Complex systems, BICS'2008, Tg.Mures, Nov.6-7, 2008, ISSN 2065-0418.

Baritz M., Cotoros D., Cristea L., Rogozea L.. "Analyze of human Body Bipedal stability for neuromotors disabilities". Bio-Inspired Computational Methods Used for difficult problems Solving. Development of Intelligent and Complex systems, BICS'2008, Tg.Mures, Nov.6-7, 2008, ISSN 2065-0418.
Baritz M., Cotoros D., Cristea L.. "Thermal Human Body Behavior Analyze During Cycling Movements". New Aspects of Heat Transfer, Thermal Engineering and Environment, WSEAS Rhodes, Greece, aug 2008, ISBN 978-960-6766-97-8, ISSN 1790-5095

Baritz M., Cotoros D., Cristea L. "Human gait analyzed by complex and interconnected system". 7th WSEAS International Conference on Applied Computer Science, Venice, Italy, november 21-23, 2007, ISBN 978-960-6766-15-2, ISSN 1790-5117;

Baritz M., Cotoros D., Moraru O., "Virtual and Augmented Reality Used to Simulate the Mechanical Device". Annals of DAAAM for 2007 \& Proceedings of the 18th International DAAAM Symposium, ISBN 3901509-58-5, pp 31, Vienna, Austria 2007

Kent Van der Graaff. "Anatomy atlas, $V^{\text {th }}$ edition, McGraw-Hill.” 1998.

Baritz M. "Study the special profiles by complementary methods". In Ed. Infomarket, Brasov, 2002.

Baritz M. "Improvement of video-audio systems and smart environment implemented in investigation methods optimization for bio-systems and person with disabilities", Research project CNCSIS A1088/2007 from Transilvania University;

Robert W., Motion Analysis and Biomechanics, http://www.laboratorium.dist.unige.it ;

Anderson F., Pandy M., Individual muscle contributions to support in normal walking, Gait and Posture 17 (2003) 159_ 169

\section{AUTHOR BIOGRAPHIES}

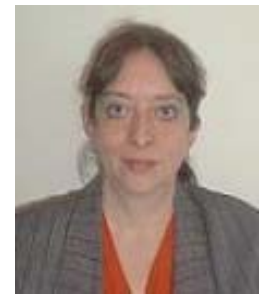

DIANA L. COTOROS was born in Brasov, Romania and went to the Transilvania University of Brasov, where she studied mechanical engineering and obtained her degree in 1986. She worked for five years for the Romanian Aircraft Company before moving in 1991 to Transilvania University of Brasov where she is now working with a research group in the field of simulation for biomechanical analysis and new implant materials identification. Her e-mail address is: dcotoros@unitbv.ro and her Web-page can be found at http://www.unitbv.ro.

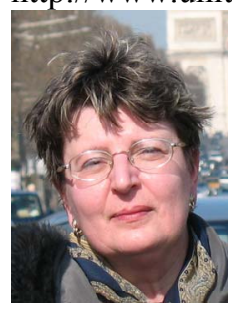

MIHAELA I. BARITZ was born in Bacau, Romania and went to Politehnica University of Bucharest, where she studied mechanical engineering and obtained her $\mathrm{PhD}$ degree in Fine Mechanics specialisation in 1997. She is now working in University Transilvania from Brasov in the field of optometry and biomechanical analysis for human behaviour. Her email address is: mbaritz@unitbv.ro and her webpage can be found at http://www.unitbv.ro. 